\title{
Blackwell, Chorin, Kailath Awarded National Medal of Science
}

On October 3, 2014, President Obama announced the recipients of the National Medal of Science for 2014. Among the ten honorees are three mathematicians: ALEXANDRE J. CHORIN, University of California Berkeley; Thomas KaIlath, Stanford University; and DAVID BLACKWELL, University of California Berkeley, honored posthumously.

\section{The Work of Alexandre Chorin}

The Notices asked Phillip Colella of Lawrence Berkeley Laboratory and James A. Sethian of the University of California Berkeley to comment on the work of Chorin. Colella and Sethian responded: "Alexandre Chorin's work is at the center of the mathematical formulation and algorithmic analysis of computer simulations in fluid dynamics over the past four decades. The wide impact of this work is due to Chorin's invention of algorithms which blend mathematical rigor with a physical insight and a deep attention to practical implementation.

"Beginning with his pioneering work nearly fifty years ago, Chorin developed the key mathematical and algorithmic ideas that underlie many of the most sophisticated, powerful computer codes in everyday practice. His work on artificial compressibility methods and projection methods provided mathematically sound approaches for imposing the key divergence-free constraint for incompressible flows. These methods are an integral part of finite difference and finite element techniques for computing flows for a host of complex applications and are ubiquitous throughout science and industry. His vortex methods are remarkably reliable techniques for computing the complex large transitory fluid structures critical to fluid mixing, wake development, and chemical transport. He was one of the pioneers of the development of high-resolution methods for gas dynamics and

DOI: http://dx.doi.org/10.1090/noti1206 combustion, in particular through his work on random choice methods.

"His groundbreaking work continues, with current work on links between statistical physics and optimal prediction of underresolved systems. Chorin has pioneered an approach to problems directed towards finding ways of predicting the evolution of systems whose behavior is not readily analyzed by present computing methods because of lack of data, uncertainty in the equations, intrinsic chaos, computational complexity, and/or multiple scales. These systems include turbulence and many biological systems and are a major challenge for computational science and engineering.

“Chorin's scientific leadership extends beyond his notable research contributions and includes his steadfast commitment to educating the next generation of applied and computational mathematicians. The many scientists he has mentored have greatly benefited from his insight, generosity, and attention."

Alexandre Chorin was born in Warsaw, Poland, in 1938. He received his $\mathrm{PhD}$ in mathematics from the Courant Institute of Mathematics at New York University in 1966 under the direction of Peter D. Lax. He served on the faculty there until 1972, when he joined the faculty at Berkeley. He is currently University Professor at Berkeley and a senior scientist at the Lawrence Berkeley National Laboratory.

His awards include the National Academy of Sciences Award in Applied Mathematics and Numerical Analysis (1989); the Norbert Wiener Prize of the AMS and the Society for Industrial and Applied Mathematics (SIAM), (2000); and the Lagrange Prize of the International Council on Industrial and Applied Mathematics (2011). He was coauthor, with J. E. Marsden, of the book A Mathematical Introduction to Fluid Mechanics (Texts in Applied Mathematics, Springer, 1993) and, with O. H. Hald, 
of Stochastic Tools in Mathematics and Science (Springer, 2013). He is the author of Vorticity and Turbulence (Springer, 1994). He is a member of the National Academy of Sciences and a fellow of the American Academy of Arts and Sciences, SIAM, and the AMS.

\section{The Work of Thomas Kailath}

The Notices asked Roger Brockett of Harvard University to comment on the work of Kailath. Brockett responded: "Tom Kailath is widely known for his work on stochastic processes, communication theory, and applications of signal processing techniques to problems in engineering. In the late 1960s he formulated the innovations conjecture, a key concept in theory of estimation of a stochastic process. The question raised involves how the sigma fields associated with a process consisting of a signal plus additive noise relates to the sigma fields associated with expected value of the process, conditioned on the knowledge of the signal plus noise. This work gave rise to a large literature extending over decades. A second area, where, again, his work cast a long shadow, involves his extension of Levenson's work on the efficient solution of finite-dimensional Toeplitz systems. These systems arise in signal processing and, in particular, in the implementation of leastsquares estimation procedures originating with Wiener. Kailath continued the investigation of fast algorithms, taking the subject in other directions involving low rank perturbations of matrices and so forth. Initially, the manufacture of integrated circuits involved the repeated exposure of photosensitive materials to light, using various masks to realize specific patterns. As the scale of the structures became smaller, the wavelength of light posed an obstacle. Kailath and his collaborators developed procedures based on Fourier analysis to overcome these limitations and worked with industry to implement practical manufacturing procedures. Kailath's $\mathrm{PhD}$ thesis at the Massachusetts Institute of Technology was devoted to the study of fading channels as they occur in communications. This work gained him a reputation of a rising star. Throughout his career at Stanford he has had a great influence on future generations through his textbooks and his work with students and postdoctoral fellows."

Thomas Kailath was born in Pune, India, in 1935. He received his $\mathrm{PhD}$ in electrical engineering from the Massachusetts Institute of Technology in 1961. He was employed at the Jet Propulsion Laboratory in Pasadena before joining the faculty of Stanford University in 1963. In 2007 he was awarded the Medal of Honor of the Institute of Electrical and Electronics Engineers (IEEE). He has also received the IEEE Jack S. Kilby Signal Processing Medal (2006) and Donald G. Fink Prize Paper Award (1996), as well as the Padma Bhushan, a high civilian award of the Government of India. He has been a fellow of the IEEE since 1970 and is a member of the US National Academy of Engineering (NAE), the National Academy of Sciences (NAS), the American Academy of Arts and Sciences (AAAS), the Indian National Academy of Engineering, and the Silicon Valley Engineering Hall of Fame.

\section{The Work of David Blackwell}

The Notices asked Peter Bickel of the University of California Berkeley to comment on the work of Blackwell. Bickel responded: "David Blackwell's National Medal of Science was awarded posthumously, apparently the very first such medal awarded after the recipient's death was known. Blackwell died on July 8, 2010, at the age of ninetyone. At the time of his death he was Professor Emeritus of Statistics and Mathematics at the University of California Berkeley. He was one of the foremost mathematical scientists in the world, contributing to mathematical statistics, probability theory, game theory, information theory, and mathematical logic. His work made a significant impact on these disciplines, as well as on economics, engineering, medicine, and other sciences.

"In statistics he is known for the Rao-Blackwell improvement scheme, for helping to lay the foundations of dynamic programming, and, with Arrow and Girshick, for applying the backward induction method to prove the fundamental theorem of sequential analysis. In a final major contribution, he asked: When can one statistical experiment be more informative than another? He defined this concept and provided a simple, necessary, and sufficient condition for experiments to satisfy this definition. This beautiful piece of mathematics has become one of the pillars of the current decisiontheoretic approach to mathematical statistics.

"In probability, Blackwell is best known for the Blackwell renewal theorem, a key tool in queueing theory. In information theory, he contributed the Blackwell channel. In game theory, he was among the first to deal with games with imperfect information. This led to his interest in mathematical logic, to which he added Blackwell games.

"Blackwell's active career, from 1941 to 1988 , was belatedly recognized with well-deserved honors: election to the National Academy of Sciences (1965) and the American Academy of Arts and Sciences (1968), with twelve honorary doctorates, and with other honors and prizes.

"His talents were recognized early but, because he was African American, the beginning of his career was harder than it should have been. To take one example of many, when he was a Fellow at the Institute for Advanced Studies, his thesis advisor, Joe Doob, had to intervene to ensure him privileges at Princeton University, which were normally granted to fellows of the Institute. 
"Blackwell was a marvelous teacher. His lucid expositions made difficult ideas seem simple and clear. His students testified to this in "A tribute to David Blackwell”, Notices 58 (2011), 912-928. With his students, colleagues, family, and friends, he was a man of exceptional kindness, wit, charm, and playfulness.

"David Blackwell deserved the National Medal of Science in his life. We celebrate the man himself and his mathematical work. We regret that he is not here with us to enjoy the honor."

David Blackwell was born in Centralia, Illinois, in 1919 and received his $\mathrm{PhD}$ from the University of Illinois at Urbana-Champaign in 1941 at the age of twenty-two. He held a fellowship at the Institute for Advanced Study in 1941-1942. He held positions at Southern University (1942-1943), Clark College (1943-1944), and Howard University (1944-1954). He went to Berkeley as a visiting professor in 1954 and became full professor in the statistics department in 1955. His book Basic Statistics (McGraw-Hill, 1969) was one of the first textbooks on Bayesian statistics. He received the John von Neumann Theory Prize in 1979, the R. A. Fisher Lectureship in 1986, and the Berkeley Citation in 1988. He served as president of the Institute of Mathematical Statistics in 1956 and vice president of the American Statistical Association in 1978. He is a member of the National Academy of Sciences (1965) and the American Academy of Arts and Sciences (1968) and an honorary fellow of the Royal Statistical Society (1976).

\section{About the Medal}

The National Medal of Science is the country's highest distinction for contributions to scientific research. According to a news release from the Office of Science and Technology Policy, "the National Medal of Science honors individuals for pioneering scientific research in a range of fields, including physical, biological, mathematical, social, behavioral, and engineering sciences, that enhances our understanding of the world and leads to innovations and technologies that give the United States its global economic edge." The National Science Foundation administers the award, which was established by Congress in 1959.

- Elaine Kehoe

\section{MANCHESTER 1824}

The University of Manchester

Faculty of Engineering and Physical Sciences School of Mathematics

\section{Turing Fellowships in Mathematics (up to 3 Posts)}

\section{$£ 34,233$ to $£ 58,172$ per annum according to relevant experience}

Named after Alan Turing, one of the most gifted and innovative researchers of the twentieth century (and was of course a member of staff of the University of Manchester), these highly prestigious fellowships are open to truly outstanding mathematicians from across the world. The fellowships are funded by a generous external donation, and are of four years duration.

Fellows will engage in research of the highest international standing in the most challenging areas of mathematics. Lecturing and administrative duties will be minimal, with teaching typically limited to one course per year on advanced material, quite possibly closely related to the fellows' research interests. Fellows will be expected to seek further funding in order to further promote their research. They will also contribute fully to the rich and diverse research life of the School of Mathematics, for example by hosting conferences in their field and supervising PhD students. Substantial funds will be available to support research and travel expenses.

Applicants will be engaging in research at the highest international standards and show the promise of becoming world leaders in their fields. Applicants will usually be outstanding early-career postdoctoral researchers, but recent doctorands of exceptional ability and potential will also be considered.

Informal enquiries can be made to Professor Peter Duck at peter.duck@manchester.ac.uk or on 01612755881.

Subject-specific enquiries can be made to Professor Peter Rowley at peter.j.rowley@manchester.ac.uk or on 01613063648.

Applications should be made online. If you are unable to apply online please request an application form by emailing hrservices@manchester.ac.uk quoting the reference number: Ref: EPS- 05368 or by calling 01612754499 (HR Services).

The University of Manchester values a diverse workforce and welcomes applications from all sections of the community.

www.manchester.ac.uk/jobs 